\title{
Kommentar zum Beitrag „Auswirkung einer künstlichen Hochwasserwelle auf den fließgewässernahen Grundwasserleiter" von Banzhaf \& Scheytt in Grundwasser (2009) 14(4): 265-275
}

\author{
Kurt-Heiner Krieger
}

Eingang des Beitrages: 27. 6.2010 / Online veröffentlicht: 24. 7.2010

(C) Springer-Verlag 2010

Die Wechselwirkung zwischen einem Vorfluter und dem mit ihm in direktem Kontakt stehenden Grundwasser ist für das hydraulische Systemverständnis von wesentlicher Bedeutung und derartige Untersuchungen daher zu begrüßen. In der vorliegenden Untersuchung sind allerdings einige Aspekte nicht zufrieden stellend berücksichtigt.

Nur bei Betrachtung der in Abbildung 1 dargestellten hydraulischen Situation wird klar, dass alle Grundwasserstände, zu jedem betrachteten Zeitpunkt, gespannte Verhältnisse in den Grundwasserleitern unter der Auelehmschicht wiedergeben, ohne das darauf eingegangen wird. Es lässt sich zwar ableiten, dass ,... eine direkte Interaktion der Systeme Bach und Grundwasser hinsichtlich der Druckverhältnisse belegt ... " ist (Seite 271), es wird im vorliegenden Artikel aber nicht darauf eingegangen, dass die Interaktion mit einer so geringen zeitlichen Verzögerung nur deshalb möglich ist, weil gespannte Verhältnisse vorliegen. Aus dem entsprechend kleinen Speicherkoeffizienten lässt sich außerdem ableiten, dass, wenn überhaupt, nur ein sehr geringes Volumen am Wasseraustausch zwischen Vorfluter und Grundwasserleiter beteiligt sein muss, um entsprechende Druckänderungen zu erzeugen.

Am 05.05.2008 liegen immer noch gespannte Verhältnisse vor, wie die Wasserstände im Auelehm belegen, und bis auf TU_03 (siehe unten) liegen die gemessenen Wasserstände der Grundwassermessstellen noch über dem Wasserstand des Baches. Es ist daher davon auszugehen, dass auch zu diesem Zeitpunkt kein „Wechsel von effluenten zu influenten Verhältnissen stattgefunden ... " hat (Seite 270). Weil wahrscheinlich immer effluente Verhältnisse vorlagen sind auch das Fehlen einer Beeinflussungder chemischen Indikatorparameter und das Fehlen von Uranin in allen Proben

Dr. K.-H. Krieger

Falkenhorst 16, 31303 Burgdorf, Deutschland erklärlich. Die vermutete horizontale Transportweite von $0,3 \mathrm{~m}$ ist zwar möglich, aber eine nicht wirklich zu begründende Größenordnung.

Die Aussagen (Seite 274), dass, bei zunehmendem Abstand vom Vorfluter und immer geringerer Mächtigkeit der Grundwasserleiter, die Druckwelle einen größeren Anstieg in der entsprechenden Messstelle bewirkt sowie die Aussage, dass mit zunehmender Entfernung der Druckimpuls zu einer Erhöhung des Druckspiegels führt, sind so nicht zu begründen. Abgesehen von den sehr, sehr geringen Absolutbeträgen (nur wenige Zentimeter) und den ungenauen Messungen mit dem Lichtlot (Messstelle TU_02 und TU_04), die schon zur Vorsicht bei derartigen Aussagen mahnen sollten, verführt anscheinend die Normierung auf den Zustand vor der künstlichen Welle (Abb. 3) zu diesen Aussagen. Bei einer Betrachtung der absoluten Werte sind derartige Rückschlüsse eigentlich nicht mehr möglich.

Der Messstellenausbau der TU_02 und TU_03 erfolgte unglücklicherweise bis an die Basis der hydraulisch trennenden Zwischenschicht. Die Abbildung 1 zeigt deutlich, dass der Bohrvorgang bis in den unteren Grundwasserleiter hinabreicht, sodass ein hydraulischer Kurzschluss über die Auflockerung der stark tonig, siltigen Zwischenschicht beim Bohrvorgang oder sogar über die Kiesfilter im Bohrloch wahrscheinlich ist. Da der Ausbau nicht detailliert dokumentiert ist, kann dies nur vermutet werden. Der abgesenkte Wasserstand am 05.05.2008 in der Messstelle TU_03, auch die geringe Absenkung an TU_02 und TU_04, beruht wahrscheinlich darauf, dass sich die Wasserstände zwischen oberem und unterem Grundwasserleiter anpassen oder ausgleichen (?) und wären damit viel zwangloser zu erklären, als z. B. mit wechselnden Aquiferparametern.

Die Berücksichtigung von Maßstabseffekten, wie sie von Sauter auf der Tagung in Tübingen diskutiert wurde, hätte wahrscheinlich auch im vorliegenden Fall zu einer befriedigenderen Erklärung der beobachteten Phänomene geführt. 